\title{
Resolving the bulk ion region of millimeter-wave collective Thomson scattering spectra at ASDEX Upgrade
}

Stejner Pedersen, Morten; Nielsen, Stefan Kragh; Jacobsen, Asger Schou; Korsholm, Søren Bang; Leipold, Frank; Meo, Fernando; Michelsen, Poul; Moseev, Dmitry; Rasmussen, Jesper; Salewski, Mirko Total number of authors:

13

Published in:

Review of Scientific Instruments

Link to article, DOI:

$10.1063 / 1.4894199$

Publication date:

2014

Document Version

Publisher's PDF, also known as Version of record

Link back to DTU Orbit

Citation $(A P A)$ :

Stejner Pedersen, M., Nielsen, S. K., Jacobsen, A. S., Korsholm, S. B., Leipold, F., Meo, F., Michelsen, P., Moseev, D., Rasmussen, J., Salewski, M., Schubert, M., Stober, J., \& Wagner, D. H. (2014). Resolving the bulk ion region of millimeter-wave collective Thomson scattering spectra at ASDEX Upgrade. Review of Scientific Instruments, 85(9), 093504. https://doi.org/10.1063/1.4894199

\section{General rights}

Copyright and moral rights for the publications made accessible in the public portal are retained by the authors and/or other copyright owners and it is a condition of accessing publications that users recognise and abide by the legal requirements associated with these rights.

- Users may download and print one copy of any publication from the public portal for the purpose of private study or research.

- You may not further distribute the material or use it for any profit-making activity or commercial gain

- You may freely distribute the URL identifying the publication in the public portal 


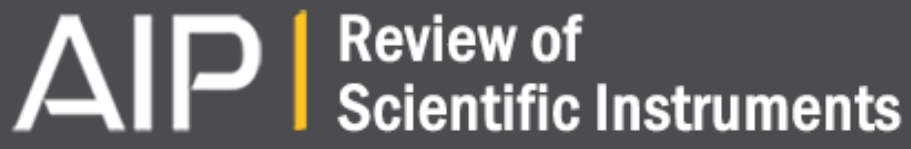

\section{Resolving the bulk ion region of millimeter-wave collective Thomson scattering spectra at ASDEX Upgrade}

M. Stejner, S. Nielsen, A. S. Jacobsen, S. B. Korsholm, F. Leipold, F. Meo, P. K. Michelsen, D. Moseev, J. Rasmussen, M. Salewski, M. Schubert, J. Stober, D. H. Wagner, and ASDEX Upgrade Team

Citation: Review of Scientific Instruments 85, 093504 (2014); doi: 10.1063/1.4894199

View online: http://dx.doi.org/10.1063/1.4894199

View Table of Contents: http://scitation.aip.org/content/aip/journal/rsi/85/9?ver=pdfcov

Published by the AIP Publishing

\section{Articles you may be interested in}

Evolution of the millimeter-wave collective Thomson scattering system of the high-field tokamak Frascati Tokamak Upgrade

Rev. Sci. Instrum. 78, 043506 (2007); 10.1063/1.2719197

Millimeter-wave backscatter diagnostic for the study of short scale length plasma fluctuations (invited)

Rev. Sci. Instrum. 77, 10E922 (2006); 10.1063/1.2235874

Frequency hopping millimeter-wave reflectometry in ASDEX upgrade

Rev. Sci. Instrum. 77, 10E915 (2006); 10.1063/1.2235206

Current fast ion collective Thomson scattering diagnostics at TEXTOR and ASDEX Upgrade, and ITER plans (invited)

Rev. Sci. Instrum. 77, 10E514 (2006); 10.1063/1.2217921

Dynamic laser alignment control for Thomson scattering on ASDEX Upgrade

Rev. Sci. Instrum. 74, 4310 (2003); 10.1063/1.1612000

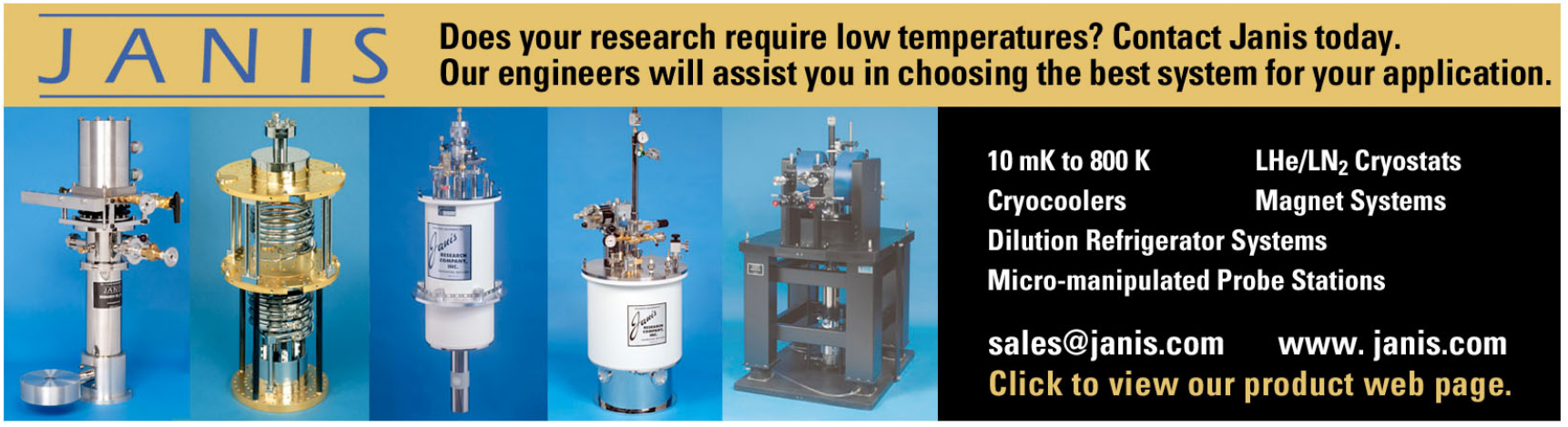




\title{
Resolving the bulk ion region of millimeter-wave collective Thomson scattering spectra at ASDEX Upgrade
}

\author{
M. Stejner, ${ }^{1, \text { a) }}$ S. Nielsen, ${ }^{1}$ A. S. Jacobsen, ${ }^{1}$ S. B. Korsholm, ${ }^{1}$ F. Leipold,${ }^{1}$ F. Meo, ${ }^{1}$ \\ P. K. Michelsen, ${ }^{1}$ D. Moseev, ${ }^{2,3}$ J. Rasmussen, ${ }^{1}$ M. Salewski, ${ }^{1}$ M. Schubert, ${ }^{2}$ J. Stober, ${ }^{2}$ \\ D. H. Wagner, ${ }^{2}$ and ASDEX Upgrade Team ${ }^{2, b)}$ \\ ${ }^{1}$ Department of Physics, Association EURATOM-DTU, Technical University of Denmark, DK-2800 Kgs. \\ Lyngby, Denmark \\ ${ }^{2}$ Max-Planck-Institut für Plasmaphysik, EURATOM-Association, Boltzmannstr. 2, 85748 Garching, Germany \\ ${ }^{3}$ Association Euratom-FOM Institute DIFFER, 3430 BE Nieuwegein, The Netherlands
}

(Received 4 April 2014; accepted 10 August 2014; published online 17 September 2014)

\begin{abstract}
Collective Thomson scattering (CTS) measurements provide information about the composition and velocity distribution of confined ion populations in fusion plasmas. The bulk ion part of the CTS spectrum is dominated by scattering off fluctuations driven by the motion of thermalized ion populations. It thus contains information about the ion temperature, rotation velocity, and plasma composition. To resolve the bulk ion region and access this information, we installed a fast acquisition system capable of sampling rates up to $12.5 \mathrm{GS} / \mathrm{s}$ in the CTS system at ASDEX Upgrade. CTS spectra with frequency resolution in the range of $1 \mathrm{MHz}$ are then obtained through direct digitization and Fourier analysis of the CTS signal. We here describe the design, calibration, and operation of the fast receiver system and give examples of measured bulk ion CTS spectra showing the effects of changing ion temperature, rotation velocity, and plasma composition. [http://dx.doi.org/10.1063/1.4894199]
\end{abstract}

\section{INTRODUCTION}

The development of robust diagnostic systems for fusion plasmas is an essential challenge when fusion experiments increase in size on the path towards realizing fusion energy as a viable energy source. The first such step is the burning plasma experiment ITER. One goal is to gain accurate knowledge of the properties of thermal and fast-ion populations in such magnetically confined fusion plasma. Collective Thomson scattering (CTS) provides such knowledge while also being a robust microwave diagnostic capable of operating under reactor relevant conditions. CTS diagnostics are based on measurements of electromagnetic probe radiation scattered off microscopic collective fluctuations, principally in the electron density. These fluctuations are created by dielectric effects of the motion of ions through the plasma, ${ }^{1}$ and CTS spectra are therefore sensitive to the ion velocity distribution with different regions of the spectra containing information about different regions in velocity-space. ${ }^{2,3}$ At relatively large frequency shifts, here roughly beyond $\pm 0.5 \mathrm{GHz}$, CTS measurements provide information about fast, non-thermal ion populations, e.g., from neutral beam heating ${ }^{4-14}$ or from fusion reactions in future experiments with burning plasmas. ${ }^{15-19}$ At lower frequency shifts, in the bulk ion region of the spectrum, CTS measurements provide information about thermal ion populations including properties such as the ion temperature and drift velocity (i.e., plasma rotation) ${ }^{20-30}$ For certain scattering geometries and plasma conditions, the bulk ion region of CTS spectra can also contain signatures of the ion cyclotron motion and of plasma waves such as the ion acoustic wave, ion Bernstein waves, and fast magnetosonic waves. Signatures of the

\footnotetext{
a) mspe@fysik.dtu.dk

b) For authors' list, see U. Stroth et al., Nucl. Fusion 53, 104003 (2013).
}

ion cyclotron motion and ion Bernstein waves are of particular interest for their sensitivity to ion density ratios. This has been used for measurements of the isotopic composition, ${ }^{30-34}$ and it could potentially be exploited for measurements of the fuel ion ratio in burning DT-plasmas. ${ }^{35,36}$ However, accessing the detailed information contained in the bulk ion region of CTS spectra requires a frequency resolution beyond what is generally achieved with the cavity filters commonly used for fast-ion measurements. Under typical conditions the ion cyclotron and ion Bernstein wave signatures create peaks in CTS spectra separated by $20-40 \mathrm{MHz}$. These effects can therefore not be resolved by the cavity filters used in fast-ion measurements, which are typically around $80-100 \mathrm{MHz}$ wide. Similarly, the Doppler shift and broadening of CTS spectra due to typical plasma rotation velocities and temperature variations are difficult to resolve properly with cavity filters. Here, we describe a new acquisition system recently added to the CTS receiver at ASDEX Upgrade ${ }^{37,38}$ (AUG) to enable measurements with frequency resolution around $1 \mathrm{MHz}$ and thereby access to the details of the bulk ion region in CTS spectra. The required frequency resolution is achieved by means of direct digitization of the CTS signal using a fast digitizer, the NI PXIe-5186, providing 8-bit dynamic range with sampling rates up to $12.5 \mathrm{GS} / \mathrm{s}$ and an analogue bandwidth of $5 \mathrm{GHz}$. Spectral information is then extracted through Fourier analysis of the time-domain data after the plasma discharge permitting flexibility in the choice of time and frequency resolution.

Below we will give an overview of the CTS systems on AUG in Sec. II and a more detailed description of the new fast CTS receiver system in Sec. III. We discuss calibration of the measured spectra in Sec. IV, and in Sec. V we go through the basic analysis steps for data from the fast receiver system including a new technique for subtraction of unwanted 
spurious signals. ${ }^{10,39,40}$ We then show examples of the measured bulk ion CTS spectra in Sec. VI and summarize our conclusions and give an outlook in Sec. VII.

\section{OVERVIEW OF CTS SYSTEMS ON ASDEX UPGRADE}

The CTS system on AUG is based on spectral measurements of frequency-shifted, near back-scattered millimeter wave probing radiation at $105 \mathrm{GHz}$. Millimeter wave based CTS is generally well suited for reactor environments, and a $60 \mathrm{GHz}$ system is being designed for ITER. ${ }^{15,41-43}$ On AUG the probing radiation is delivered by a dual frequency gyrotron normally used for electron cyclotron resonance heating $(\mathrm{ECRH})$ at $140 \mathrm{GHz}$. The probing gyrotron is part of the ECRH-2 system on AUG, ${ }^{44-46}$ which is comprised of four gyrotrons referred to here as gyrotrons 5-8 (gyrotrons 1-4 comprise the ECRH-1 system on AUG). The gyrotrons have separate transmission lines and launching antennae, which are mounted in close proximity above and below the equatorial midplane on the low-field side on the tokamak as illustrated in Fig. 1.

The launching antennae are steerable and CTS measurements can in principle be based on probing radiation from any of the four gyrotrons, which provides flexibility in the choice of scattering geometry. For the measurements presented here, we used probing radiation from gyrotron 7 operated at $105 \mathrm{GHz}$ and with an output power of $600 \mathrm{~kW}$. At $105 \mathrm{GHz}$ and with the standard $2.5 \mathrm{~T}$ on-axis magnetic field in AUG, the fundamental and second harmonic resonances of the probing radiation are located near the edges or outside the plasma

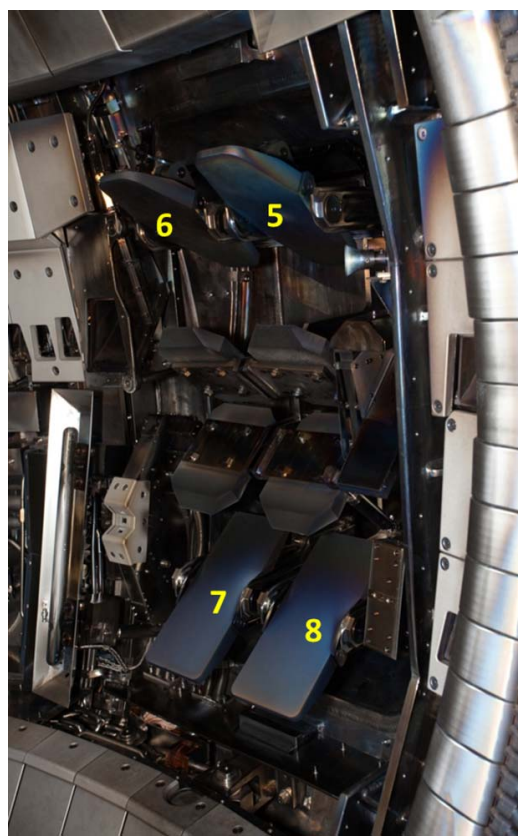

FIG. 1. The launching mirrors of the ECRH-2 system on AUG. During ECRH operation the mirrors are used to launch radiation from gyrotron 58 as labeled in the picture. For the measurements presented here, gyrotron 7 provided the probing radiation while the mirrors and transmission lines for gyrotron 6 and 5 were used to view the plasma with receiver $\mathrm{A}$ and $\mathrm{B}$, respectively. on either side. This choice of probing frequency thus minimizes refraction and absorption of the radiation as well as the level of background signals in the relevant frequency range. To further reduce refraction and absorption in the plasma, the probing radiation is polarized for $\mathrm{O}$-mode. To enable subtraction of the remaining background, which originates mainly from electron cyclotron emission (ECE) near the plasma edge, the gyrotron power is modulated on/off - usually with onperiods of $2 \mathrm{~ms}$ followed by off-periods of $3 \mathrm{~ms}$.

The CTS system on AUG includes two separate receiver systems based on the standard filter bank technique used for fast-ion measurements. These can be operated simultaneously and provide similar though not identical spectral coverage. The fast acquisition system can be coupled to either of the two receivers to enable measurements with high frequency resolution on that receiver. As we shall see below, data from the filter bank receivers are also useful for background subtraction and validation of data from the fast acquisition system. To place the fast acquisition system in its proper context, we will therefore briefly discuss the two standard filter bank receivers.

For ease of reference, we will here refer to these systems as receiver A and B. Receiver A was the first to be installed on AUG, and this system has been described in some detail elsewhere. ${ }^{10,47,48}$ The intermediate frequency (IF) parts of receiver $\mathrm{B}$, including the mixing stage and filter bank, were recently installed on AUG having previously been used for CTS measurements on TEXTOR. Descriptions of these elements of receiver B can therefore also be found elsewhere. ${ }^{49-51}$

Each CTS receiver resolves scattered radiation picked up with one of the ECRH antennae not used to launch the probing radiation. The radiation is transmitted to the receivers via the corresponding ECRH waveguides, which are reserved for this purpose during CTS measurements. Here, receiver A was coupled to the waveguide of gyrotron 6 using a movable mirror in the matching optics unit (MOU) box of that gyrotron. ${ }^{47}$ Receiver B was coupled to the waveguide of gyrotron 5 using a waveguide switch, which can couple the waveguide either to the gyrotron (for standard operation with ECRH heating) or to the CTS receiver. As illustrated in Figs. 1 and 2, the receivers were thus coupled to two antennae located next to each other above the mid-plane and displaced horizontally from each other. The antenna used to launch the probing radiation from gyrotron 7 was located vertically below the antenna of gyrotron 6, which was coupled to receiver A.

While they differ in the details, the two receiver systems are conceptually very similar. As illustrated in Figs. 3 and 4, they both include a pair of notch filters centered on the probing frequency. These are followed by a heterodyne down-mixing stage and a filter bank with cavity filters and diodes connected to NI-4472 ADCs, which provide 24-bit dynamic range at a sampling rate of $100 \mathrm{kS} / \mathrm{s}$. Each notch filter provides about $60 \mathrm{~dB}$ attenuation in a roughly $250 \mathrm{MHz}$ wide frequency range centered around the probing frequency. This protects the receiver electronics against stray probing radiation, but it also suppresses the CTS signal in that range leaving only a relatively weak signature of the gyrotron frequency. During the gyrotron start-up and shut-down phases the probing frequency can drift outside the stopbands of the 


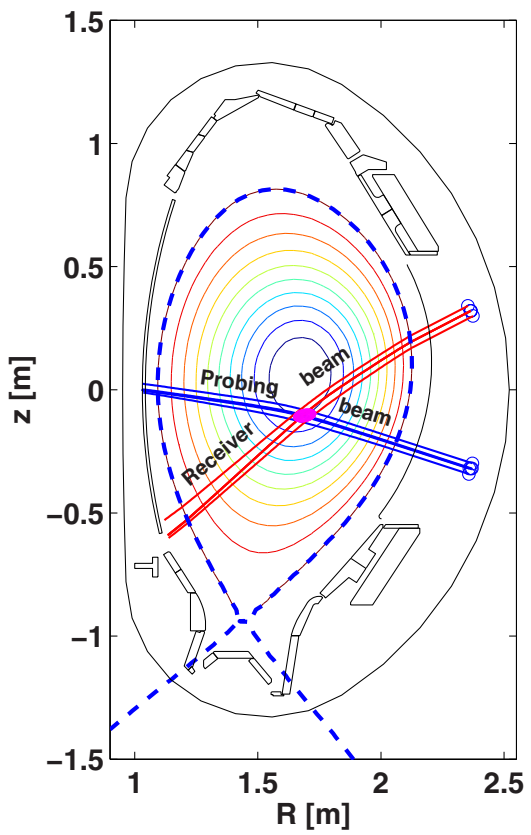

FIG. 2. A poloidal projection of the CTS beam geometry in AUG discharge 29600. Beam patterns representing the incident and received scattered radiation were calculated by ray tracing. Magnetic flux surfaces are indicated by the nested contours and the separatrix by the thick dashed line. The probing radiation was launched from the gyrotron 7 launching mirror below the midplane on the low field side of AUG. Scattered radiation was picked up using the mirror and transmission line of gyrotron 5 located above the equatorial midplane on the low field side. The CTS measurement is localized in the measurement volume defined by the beam overlap indicated by the magenta ellipsoid. notch filters. At those times a voltage controlled variable attenuator (VCVA in Figs. 3 and 4) is used to provide a further $30 \mathrm{~dB}$ attenuation over the entire bandwidth. The signal is then down converted by means of a local oscillator and a mixer and fed to the filter bank. There the signal is split into individual frequency bands using a sequence of power dividers and bandpass filters terminated by detector diodes. The voltage at the diodes, representing the power, is then measured with the NI-4472 ADCs. In receiver A, the signal is initially split using an active triplexer unit with individually shielded compartments for each frequency band. This receiver also includes bypass channels, which monitor the overall signal level before it is split as well as the signal level in each of the main frequency ranges. A detailed characterization of receiver A was given in a recent paper in this journal. ${ }^{48}$ In receiver $\mathrm{B}$, the signal is initially split by means of a triplexer and a diplexer, and there are no bypass channels.

For measurements with the fast acquisition system, power dividers were inserted in the main bypass channel of receiver $\mathrm{A}$ and after the diplexer in the mid-frequency line of receiver B. In both receivers, the signal is then fed through a band pass filter selecting a roughly $3 \mathrm{GHz}$ wide frequency range around the probing frequency. The signal in the selected frequency range is then amplified before it is fed to the fast acquisition system described in Sec. III. The fast acquisition system can thus be connected to both receivers using similar interfaces. For the measurements discussed below the system was mounted on receiver $B$.

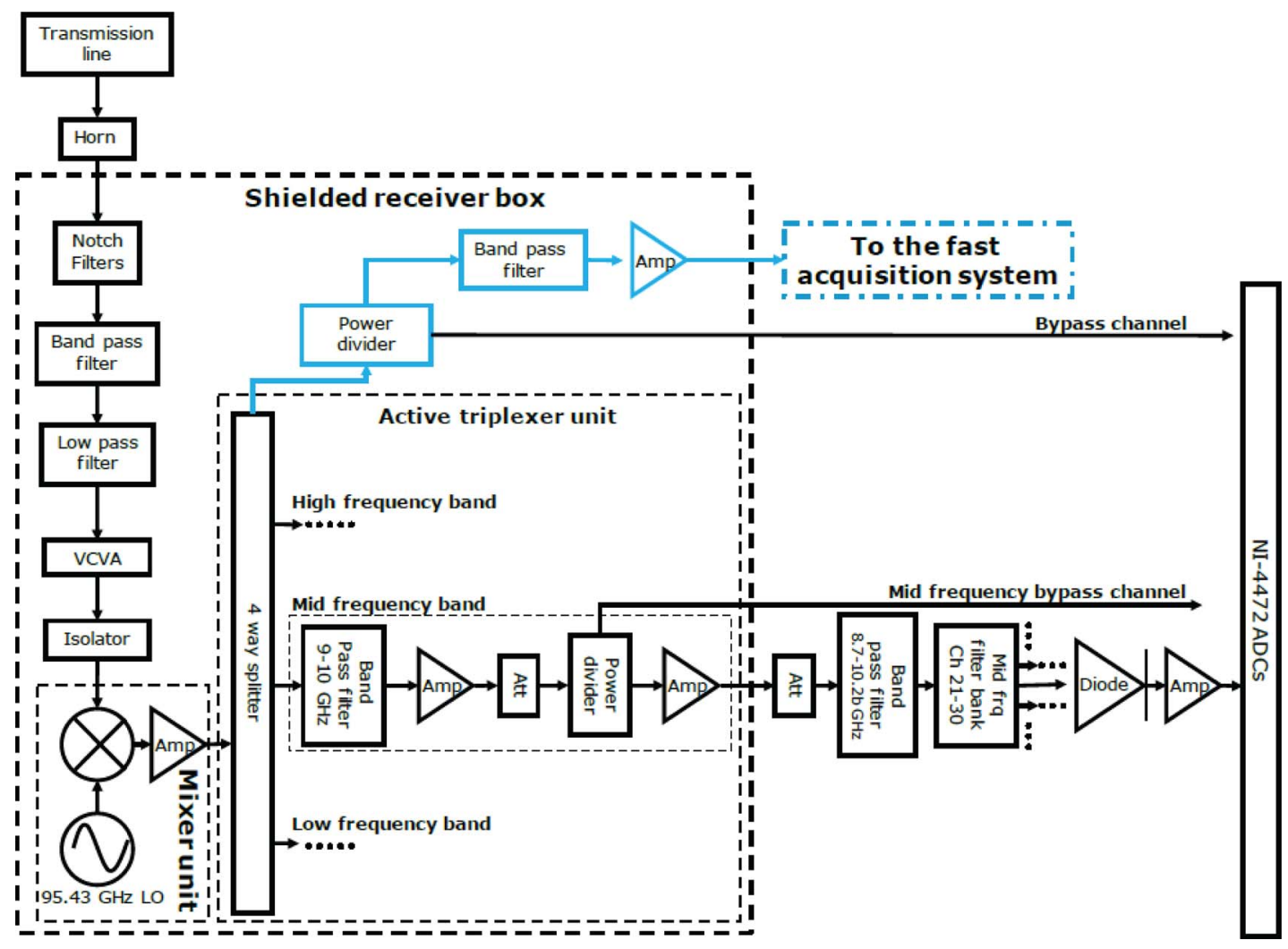

FIG. 3. A block diagram illustrating the design of receiver A. Dashed lines represent metallic shielding compartments. The component sequences in the highand low-frequency bands are analogous to those of the mid-frequency band. Such repeated sequences of components are represented with dotted lines. Parts related specifically to the fast acquisition system are marked in light blue. 


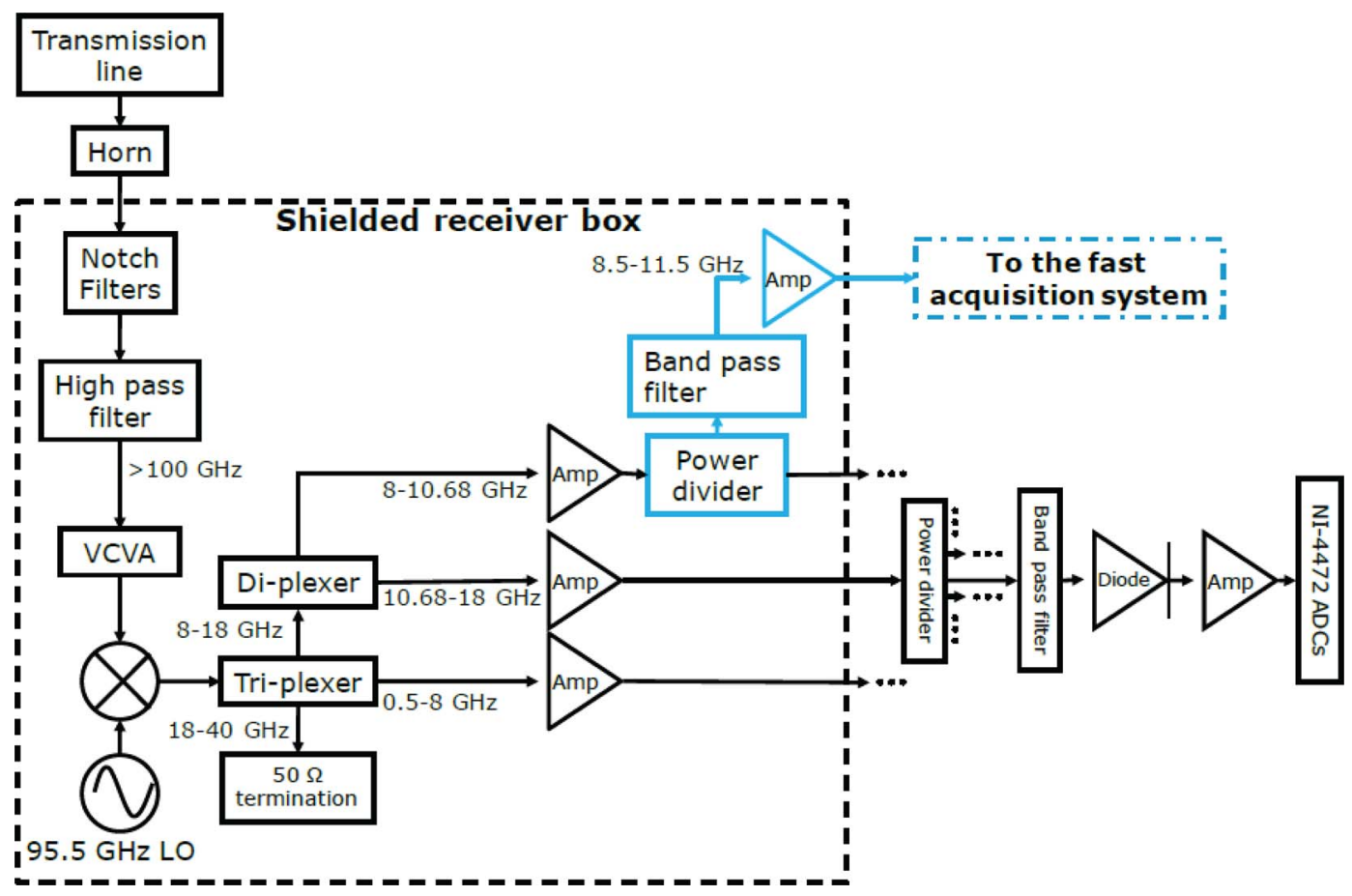

FIG. 4. A block diagram illustrating the design of receiver B. Dashed lines represent metallic shielding compartments. Repeated sequences of components are represented with dotted lines. Parts related specifically to the fast acquisition system are marked in light blue.

\section{FAST ACQUISITION SYSTEM FOR CTS}

The fast acquisition setup is illustrated in Figure 5. The system is mounted in an NI-PXIe 1065 chassis and includes a stepwise variable attenuator, an isolator, a mixing stage, an amplification stage, a low-pass filter, and an NI PCIe-5186 ADC sampling the down converted signal. The stepwise variable attenuator has four discrete levels providing between 10 and $28 \mathrm{~dB}$ attenuation. This was included to enable the system to span the full range of signal levels encountered in experiments and during testing and calibration without saturating or damaging the equipment. For calibration purposes, each attenuation level was characterized using a network analyzer. The bandwidth limited IF signal from the standard filter bank receiver is further down converted by means of a mixer and a local oscillator with a fixed frequency of $8.13 \mathrm{GHz}$. With the system connected to receiver B as in the present experiments, the $105 \mathrm{GHz}$ probing frequency was thus down converted to $1.32 \mathrm{GHz}$ and the signal passed to the ADC then represented an original frequency range from 104.0 to $106.2 \mathrm{GHz}$. So effectively the ADC saw a signal corresponding to an approximately $2 \mathrm{GHz}$ wide band centered roughly on the probing frequency. Digitizing the down converted signal is thus well within the capabilities of the NI PCIe-5186 ADC given its $5 \mathrm{GHz}$ analogue bandwidth and sampling rate ranging up to $12.5 \mathrm{GS} / \mathrm{s}$.

The NI PCIe-5186 has an on-board memory of 1 GB and for the present experiments it was operated with a sampling rate of $6.25 \mathrm{GS} / \mathrm{s}$ permitting a total acquisition time of $160 \mathrm{~ms}$ during each plasma pulse. Thus, it is presently not possible to measure continuously during an entire AUG plasma pulse, so the limited memory and acquisition time must be allocatedto times of particular interest for each experiment. For this

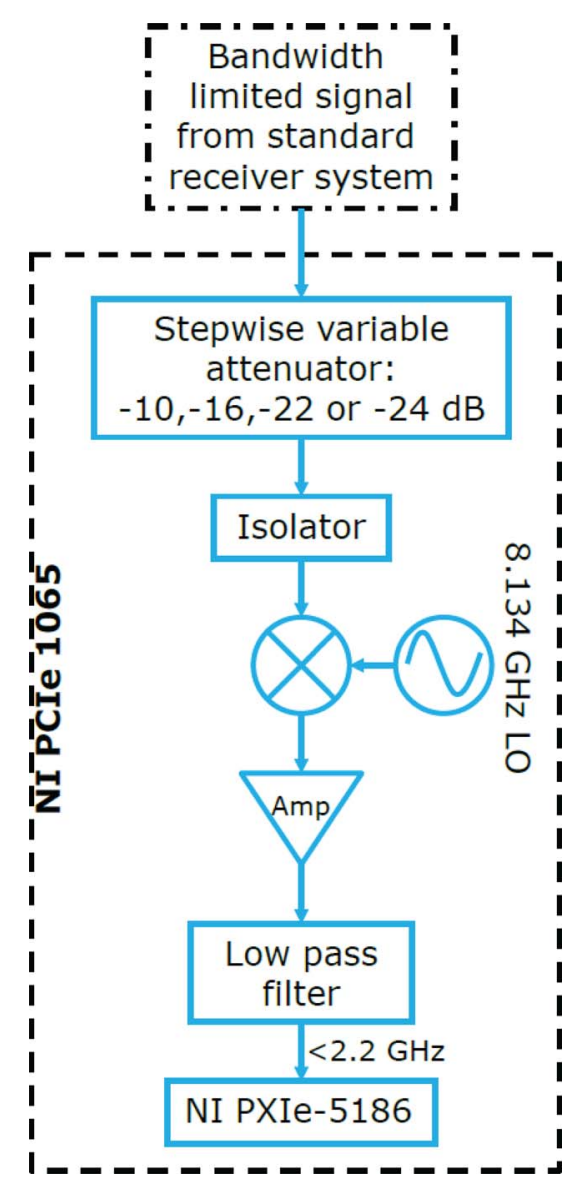

FIG. 5. A block diagram illustrating the fast acquisition system. The system is mounted in an NI-PCIe 1065 indicated by the dashed line. The system is thus movable and can be connected to any of the two filterbank receiver systems. 
purpose, individual data acquisitions by the ADC are triggered by an externally generated trigger signal. The timing of these triggers can be adapted to the experiment as needed and synchronized to the gyrotron pulses, which are also triggered independently by a similarly adaptable signal. With this system both the gyrotron pulses and the individual acquisitions can be triggered at the most appropriate time for each experiment. A reasonable signal-to-noise level can typically be achieved with data acquisitions of $0.5 \mathrm{~ms}$ for each selected gyrotron pulse and $0.25 \mathrm{~ms}$ in the subsequent gyrotron-off period, which permits a total of 213 gyrotron pulses to be sampled.

Spectral information is extracted after the experiment through Fourier analysis using the standard FFT tools available in Matlab. The time and frequency resolution are then adjustable as they depend on the length of the chosen Fourier window. For analysis of the data discussed below, we used a window of $n_{\mathrm{FFT}}=2^{12}=4096$ data points with a Hann window function. This window length corresponds to a time resolution of $0.6 \mu \mathrm{s}$ and a frequency resolution of $1.53 \mathrm{MHz}$. The time resolution is subsequently reduced by averaging over an appropriate time interval to get a reasonable signal-tonoise ratio. For comparison with CTS models and inference of plasma parameters, we generally use data averaged over at least one gyrotron pulse giving an effective time resolution in the millisecond range depending on the chosen gyrotron modulation rate.

\section{CALIBRATION}

To adjust for the instrument function, we perform a linear calibration based on the difference between the emission spectra observed in alternate measurements of two blackbody sources at different temperatures. For this purpose, we use two microwave absorbers (Eccosorb material) held at room temperature and cooled with liquid nitrogen, respectively. A moveable chopper mirror is used to direct the receiver beam into a bucket lined with Eccosorb and filled with liquid nitrogen. The chopper mirror and the liquid nitrogen box are located along the transmission line immediately outside the receiver box. A different piece of Eccosorb at room temperature is inserted in the transmission line and observed when the chopper mirror does not intercept the receiver beam.

Figure 6 shows an example of such measurements. Here, we used the lowest $(10 \mathrm{~dB})$ setting of the stepwise variable attenuator in the fast acquisition system. The data represent a total acquisition time of $48 \mathrm{~s}$, half from each source. The observed difference in signal intensity and the resulting signal-to-noise ratio depends strongly on frequency. In the frequency range covered by the notch filters, approximately 1.2-1.45 GHz after down conversion, the signal is entirely suppressed and the difference cannot be observed. Outside the notch filters the difference is distinguishable above the noise, and the signal-to-noise ratio for the difference reaches a range between 40 and 70 .

The strength of this calibration method is that it is independent of other diagnostics and of any plasma operation. Calibration can thus be repeated at almost any time (typically just before or after an experiment) and for any length of time
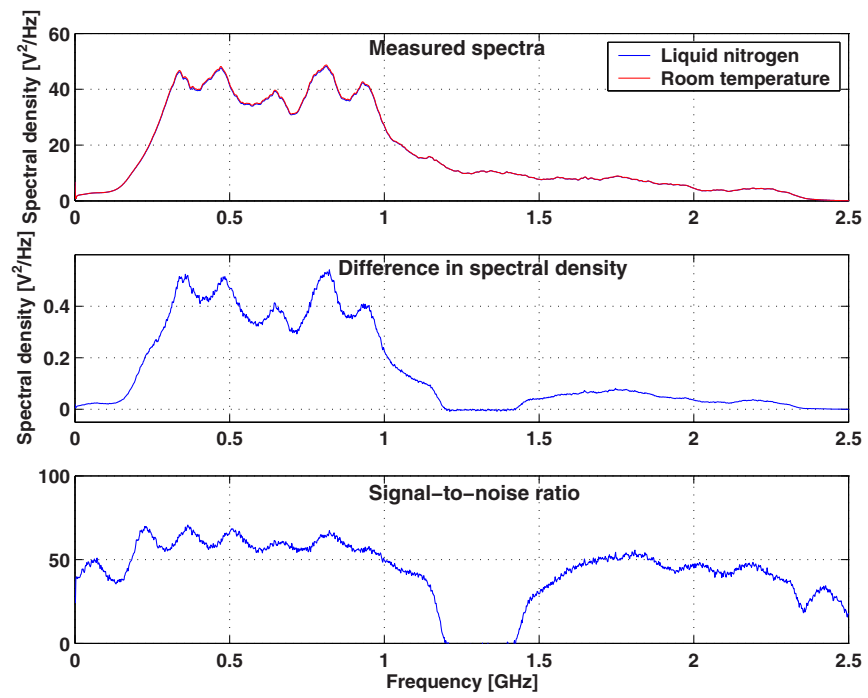

FIG. 6. Examples of spectra used for liquid nitrogen calibration. The top panel shows the spectral power density measured from blackbody sources at room temperature and cooled with liquid nitrogen, respectively. The difference between the spectra cannot be distinguished on this scale and is shown separately in the middle panel. The lower panel shows the signal-to-noise ratio for the difference over the entire bandwidth of the measured spectra. The effect of the notch filters are seen at frequencies of approximately 1.2$1.45 \mathrm{GHz}$. There the spectra are indistinguishable and entirely dominated by receiver noise.

up to the constraints set by the size of the available memory. The signal-to-noise ratio can therefore in principle be improved simply by taking more data, which would in particular help improve the final data quality in the frequency range just outside the stopband of the notch filters. The data in Fig. 6 represent $3.12 \times 10^{11}$ samples corresponding to a data set of $770 \mathrm{~GB}$ when stored in HDF5 format. While this is somewhat cumbersome with the current analysis setup, using a standard hard-drive and a single processor for the FFT calculation, there are plenty of hardware options available which could handle greater data sets to improve the signal-to-noise ratio for calibration purposes.

The main challenge with this calibration method is that the signal levels are orders of magnitude below those encountered during CTS experiments, which places stringent requirements on the linearity of the system and necessitates the use of the variable attenuator. The calibration is based on a difference in spectral power density between the hot and cold source of approximately $19 \mathrm{meV}$ whereas the spectral power densities encountered during CTS measurements reach levels in the range 10-500 eV. This range can be covered by varying the dynamic range of the digitizer itself, which is variable between $0.1 \mathrm{~V}$ and $1 \mathrm{~V}$ (peak-to-peak), and by using the lowest setting of the variable attenuator for calibration and the highest or second highest setting for CTS experiments. The calibration curve is then adjusted numerically to the relevant setting of the variable attenuator using the measured attenuation levels. Similarly, the effects of the diamond window between the ECRH waveguides and the vacuum vessel are also included numerically in the calibration based on a theoretical calculation of the small amount of reflection caused by this window. ${ }^{44}$ Effects of absorption in the high-power ECRH waveguides are not expected to change the spectral shape 
significantly across the narrow frequency range monitored here and are neglected.

The final calibration obtained from liquid nitrogen measurements relates the spectral density, $P_{\mathrm{V}^{2} / \mathrm{Hz}}$, of the potential measured by the ADC (in $\mathrm{V}^{2} / \mathrm{Hz}$ ) to the spectral power density, $P_{\mathrm{e} V}$, of the received radiation (in $\mathrm{eV}$ ). It is given by

$$
\begin{aligned}
P_{\mathrm{eV}}(v) & =a(v) P_{\mathrm{V}^{2} / \mathrm{Hz}}(v)+b(v), \\
a(v) & =\left(\frac{\Delta T}{\Delta P_{\mathrm{V}^{2} / \mathrm{Hz}}(v)}\right)\left(\frac{A_{\mathrm{n}}(v)}{A_{1}(v)}\right) A_{\mathrm{W}}(v) .
\end{aligned}
$$

Here, $v$ is the frequency, $\Delta T=19 \mathrm{meV}$ is the temperature difference between the blackbody sources, and $\Delta P_{\mathrm{V}^{2} / \mathrm{Hz}}$ is the difference, shown in the middle panel of Fig. 6, between the spectral densities measured by the ADC. $A_{\mathrm{n}}$ is the measured attenuation from the variable attenuator at the relevant attenuation level, $n$, with $n=1$ corresponding to the lowest $(10 \mathrm{~dB})$ setting, etc. $A_{\mathrm{W}}$ is the theoretically calculated attenuation of the signal due to reflection in the diamond window between the waveguides and vacuum vessel. Results for the calibration coefficients, $a(v)$, are shown in Fig. 7. The offset, $b(v)$, can also be obtained from the calibration measurements, but in CTS measurements it cancels when the background is subtracted and it is not shown here. From Figs. 6 and 7, we note that the system is less sensitive at frequencies above than below $1 \mathrm{GHz}$. However, we also note that this leads to no significant degradation of the signal-to-noise ratio: see the lower panels of Fig. 7 and Fig. 11 in Sec. V below. This indicates that the signal is still well resolved by the acquisition system at those frequencies, and that the dominant source of noise in the system is located before the (presently unidentified) component with reduced transmission above $1 \mathrm{GHz}$.

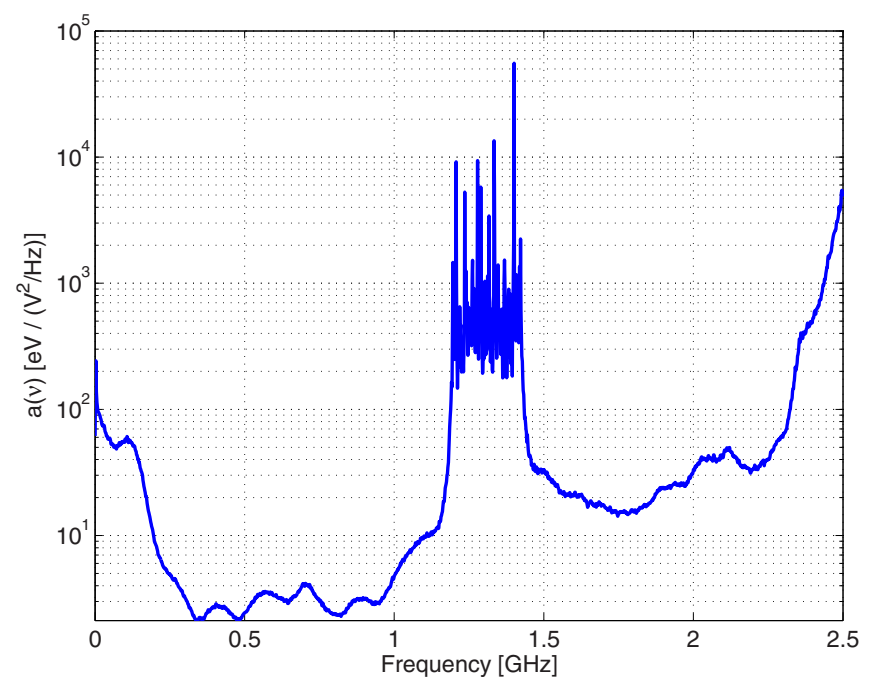

FIG. 7. The calibration coefficients, $a(v)$, as defined in Eq. (1), obtained from liquid nitrogen measurements. In the region covered by the notch filters, around 1.2-1.45 GHz, the calibration coefficients are dominated by noise because the relatively weak signals used for calibration cannot pass the notch filters. That region is therefore excluded in the analysis below.

\section{BASIC DATA ANALYSIS}

Figure 8 shows an example of uncalibrated, raw spectra obtained with the fast acquisition system during a CTS experiment in AUG discharge 29600. The top panel shows a spectrogram for a narrow time frame containing two gyrotron pulses. Here, the data are binned to give a time resolution of $50 \mu \mathrm{s}$ in this spectrogram (as described below the data will later be re-binned for a final time resolution of $8 \mathrm{~ms}$ ). At this particular time, the gyrotron was modulated in a $25 \%$ duty cycle with $2 \mathrm{~ms}$ on-periods and $6 \mathrm{~ms}$ off-periods. For every gyrotron pulse, the fast acquisition system was triggered for three acquisition periods of $0.25 \mathrm{~ms}$ each: two acquisitions during the gyrotron-on periods and one shortly after the gyrotron turn-off. This unequal allocation of acquisition time improves the final signal-to-noise ratio when the CTS signal dominates over the background, as is the case for measurements of the bulk-ion region of CTS spectra on AUG. As illustrated in Fig. 2 and below in Fig. 10, the measurements were localized in the measurement volume at the intersection of the probe and receiver beams. The position of the measurement volumes was found by ray tracing. The measurement volume was approximately $10 \mathrm{~cm}$ across and was centered on $(R, z)=(1.73$ $\mathrm{m},-0.11 \mathrm{~m})$ corresponding to a normalized poloidal flux coordinate of 0.37. The lower panel in Fig. 8 shows examples of individual spectra (at the $50 \mu$ s time resolution) obtained during gyrotron-on and -off periods, respectively. During the gyrotron-on period the signal is elevated, indicating the presence of a CTS signal, at frequencies outside the region covered by the notch filter stopbands $(1.2-1.45 \mathrm{GHz})$. Inside the notch filter region only the gyrotron frequency can be identified, and this is only possible before the calibration is applied as the calibration is itself entirely dominated by noise in that frequency range. Depending on the duty cycle, the gyrotron frequency drifts slightly by a few $\mathrm{MHz}$ during each gyrotron pulse and across the course of a plasma discharge $e^{44,51,52}$ due to thermal expansion of the gyrotron cavity. In order to monitor and adjust for this drift, the first step in the analysis is therefore to identify the gyrotron frequency in the uncalibrated data for each $50 \mu$ s time frame. The calibration is then applied and the background measured in the corresponding gyrotron-off period is subtracted. After background subtraction we adjust for the gyrotron frequency drift by selecting a fixed frequency range centered on the gyrotron frequency for each $50 \mu$ s time frame. Subsequent analysis is then performed in terms of the frequency shift relative to the probing frequency.

On AUG it has been found that the background in CTS measurements consists of two parts that must be dealt with differently. ${ }^{39}$ The dominant part of the background is the standard ECE radiation from the plasma. To subtract this, the gyrotron power is modulated on/off and the ECE background measured during the gyrotron-off periods is subtracted, as discussed above. The top panel in Fig. 9 shows an example of calibrated data from the fast acquisition system (AUG pulse 29600, the first gyrotron pulse in Fig. 8) measured with the gyrotron on and off. Here, the bulk ion CTS spectrum dominates the gyrotron-on spectrum at frequency shifts within $\pm 300 \mathrm{MHz}$ (the precise limits vary depending on plasma 

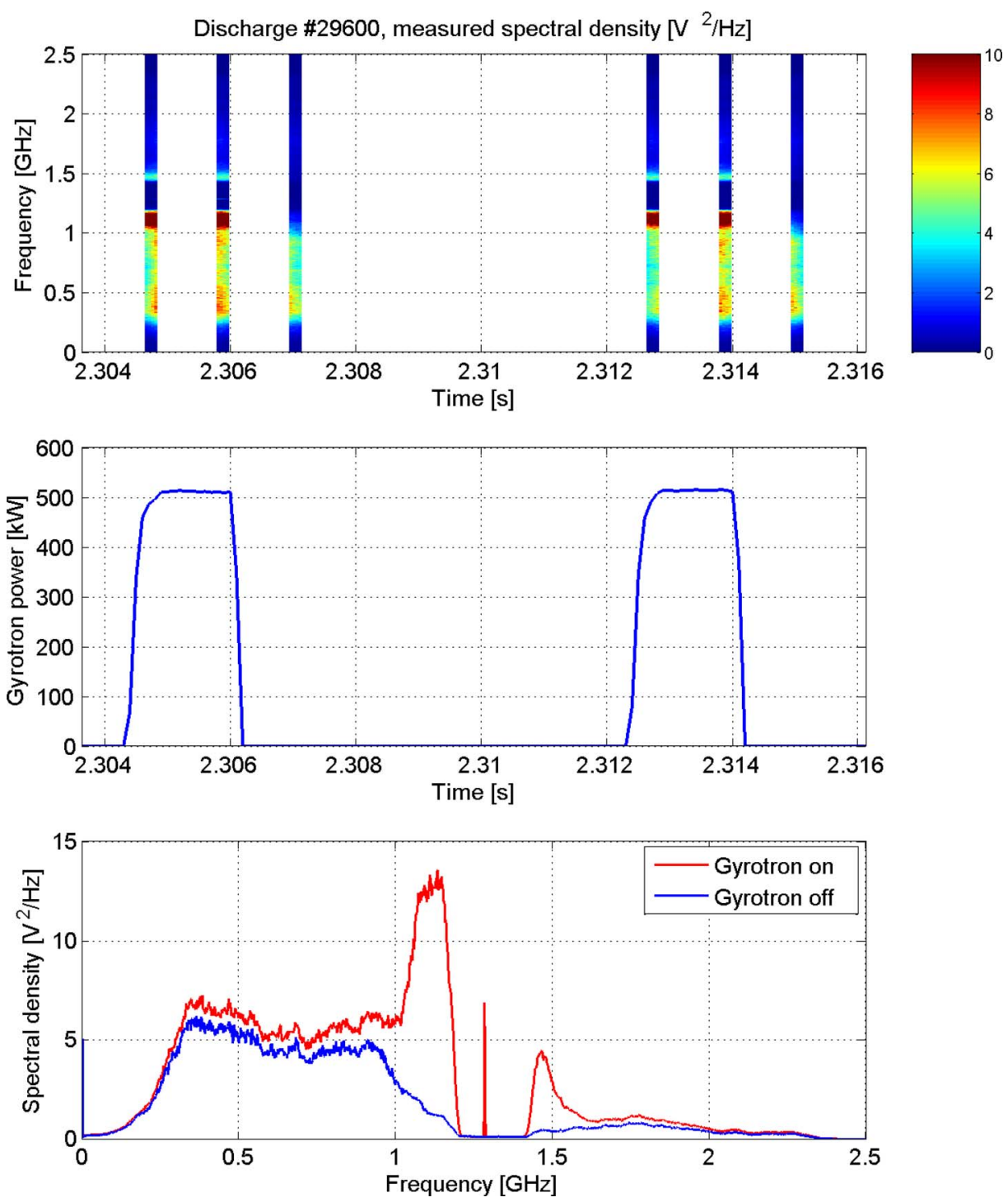

FIG. 8. An example of raw, uncalibrated spectra measured in discharge 29600. The top panel shows a spectrogram of the measurements from a narrow time range containing the 2 gyrotron pulses illustrated in the middle panel, which shows a time trace of the gyrotron power. In the top panel, the spectrogram is shown for a time resolution of $50 \mu \mathrm{s}$. Two acquisitions of $0.25 \mathrm{~ms}$ were triggered during each gyrotron pulse and one immediately after. The lower panel shows individual spectra (at $50 \mu \mathrm{s}$ time resolution) with the gyrotron on and off, respectively. During the gyrotron-on period, scattering enhances the spectral power density outside the notch filter region, and the gyrotron line can be seen above the noise in the notch filter region.

temperature among other things). Beyond that range the spectrum is dominated by the ECE background, but in addition the gyrotron-on spectrum is found to contain a small, spectrally flat offset of a few $\mathrm{eV}$ extending to large frequency shifts - even beyond the range of the fast acquisition system and beyond the range that can plausibly be explained by effects of fast ions. The origins of this component are presently not fully understood, but it is known that it does not originate from the CTS measurement volume, defined by the region of overlap between the probe and receiver beams, since it can be measured when no such overlap is present. Leading hypotheses for the mechanism behind this so-called spurious emission include gyrotron noise, absorption and re-emission of probing radiation, as well as parametric decay of the probing radiation. However, for CTS measurements, our main concern is to subtract the spurious emission in order to extract the CTS spectrum originating from the beam overlap. While this makes a relatively small difference for the type of bulk ion measurements discussed here, it is important for fast-ion measurements relying on much smaller signal levels at larger frequency shifts. ${ }^{39,40}$ For this purpose, the receiver mirrors for the two CTS systems are oriented with identical toroidal and poloidal viewing angles. Given the horizontal displacement of the two receiver mirrors, this means that the receiver beams are parallel, and that only the beam for receiver B overlaps with the probing beam - see Fig. 10 for an illustration.

We then refer to the measurements with receiver A (having no overlap with the probing beam) as passive measurements and to those by receiver B (with overlap) as active measurements. Although receiver A views the plasma passively it still sees the spurious emission as illustrated in Fig. 9. With parallel receiver beams, it has further been found that the spurious emission spectrum seen by receiver A corresponds closely to that measured by receiver $\mathrm{B}$ when neither receiver 

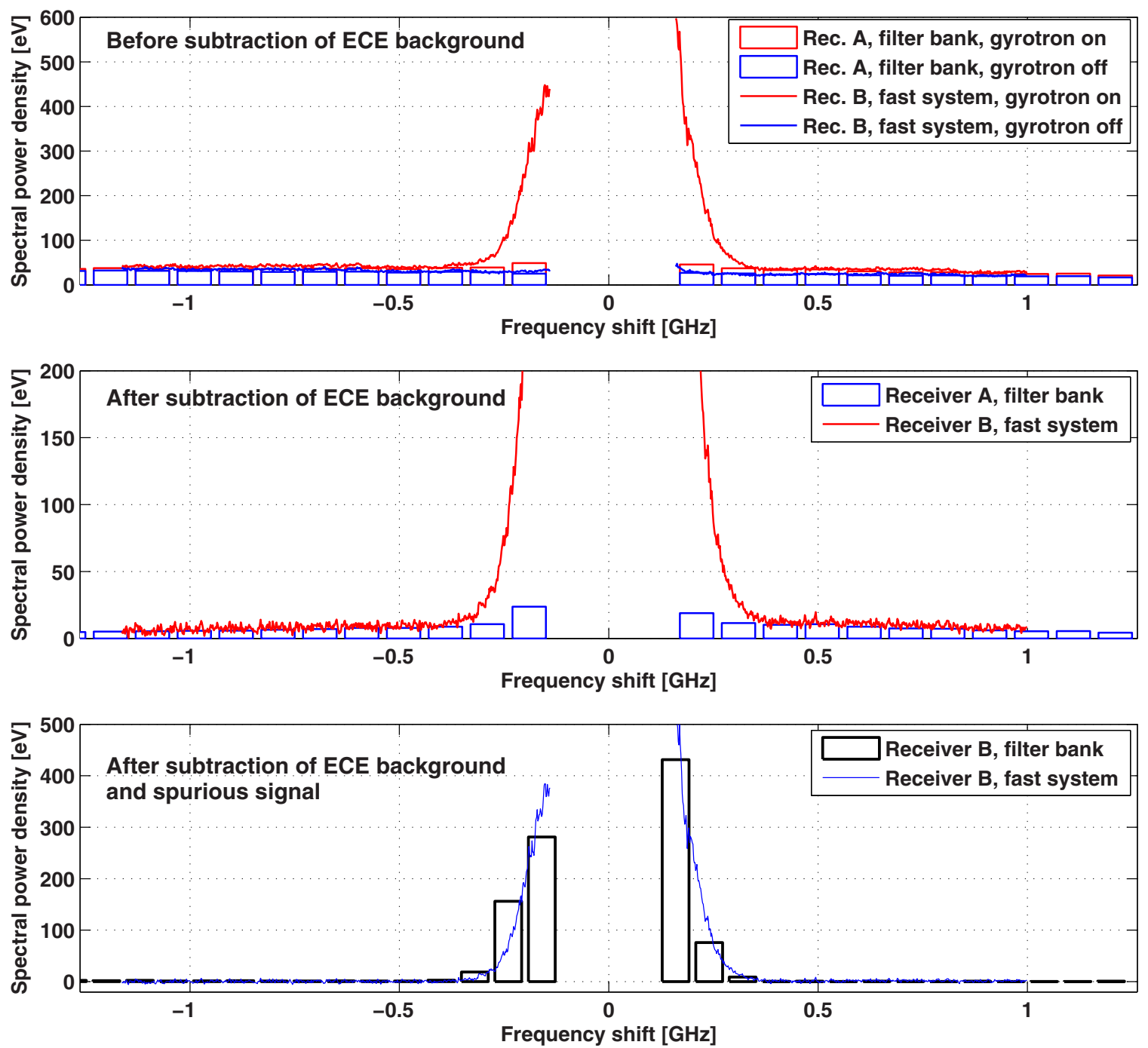

FIG. 9. An example of calibrated data measured in AUG discharge 29600. Here and in figures below, data from the frequency range affected by the notch filters are excluded as they are extremely noisy can not be accurately calibrated. The top panel shows passive measurements during gyrotron-on and -off periods with the filterbank in receiver A and active measurements from the fast acquisition system mounted on receiver B. CTS signal is clearly seen above the background during the gyrotron-on period in the active measurements. In addition to the ECE background observed with the gyrotron off there is also a low level of secondary emission seen with the gyrotron on in both the passive filterbank measurements and the active measurements with the fast acquisition system. Both the ECE background and the secondary emission must be subtracted to obtain the CTS spectrum shown in the lower panel as seen by both the filterbank on receiver B and by the fast acquisition system.

has an overlap with the probing beam. For CTS measurements, we therefore subtract the spurious emission spectra measured with the passive view by receiver A from the active measurements with receiver $\mathrm{B}$, which measures the sum of CTS and the spurious emission. More detailed descriptions of background subtraction using this dual-receiver technique for fast-ion measurements have been given elsewhere. ${ }^{39,40}$ For bulk ion measurements, the passive measurements with receiver A should ideally be resolved at the same frequency resolution as the active measurements with the fast acquisition system on receiver B. However, at present there is no fast acquisition system installed on receiver A, so we interpolate and subtract the spurious emission spectra measured with the filter bank on receiver A. The spurious emission depends only weakly on frequency shift (i.e., it is spectrally flat). Inaccuracies due to the interpolation process are therefore expected to be small relative to other components in the spectra and relative to other uncertainties.
The lower panel of Fig. 9 shows an example of the final CTS spectrum obtained after calibration, adjustment for gyrotron drift, averaging over a gyrotron pulse, subtraction of ECE background, and subtraction of spurious emission. The CTS signal is clearly seen at frequency shifts within $\pm 400 \mathrm{MHz}$ (depending on temperature), while the spectrum now oscillates around zero at frequency shifts beyond that range. Also, the spectrum is consistent with similarly treated simultaneous measurements with the filter bank on receiver $\mathrm{B}$.

To estimate the statistical uncertainty in the measured spectra, we evaluate the noise level in the data measured during the plasma discharge and in the calibration data for each receiver system. For this purpose, we calculate the standard error on the mean spectral power density for each type of measurement based on the level of temporal fluctuations. The top panel in Fig. 11 shows the contributions to uncertainty in the spectrum shown in Fig. 9 from noise in the filter bank on 


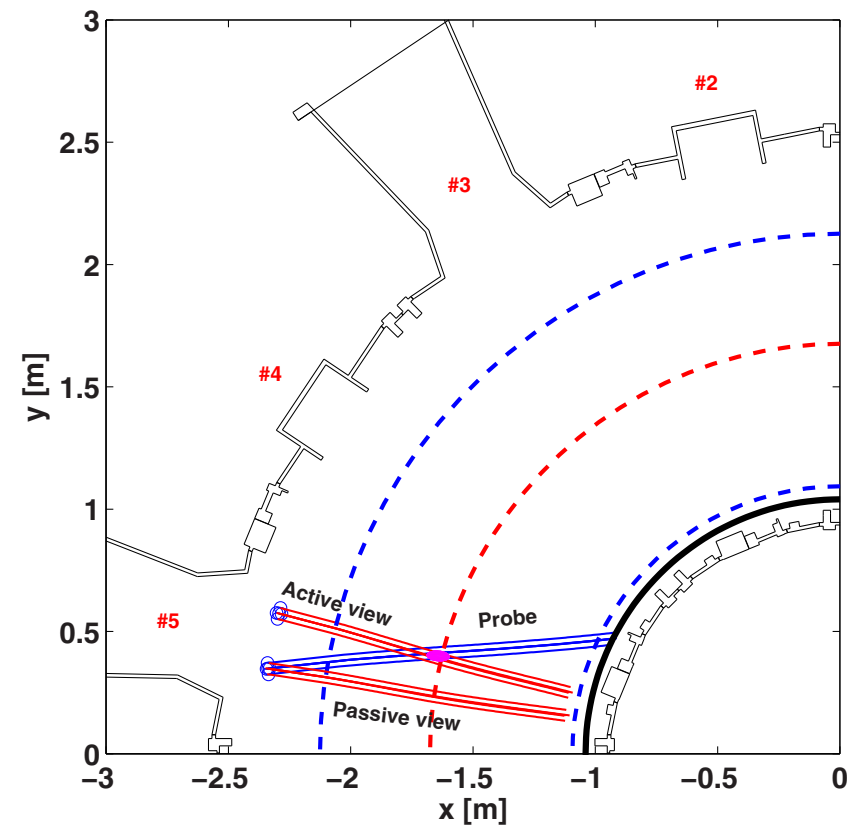

FIG. 10. A top view of the beam geometry in AUG discharge 29600 illustrating the use of parallel receiver beams to monitor both the spurious emission, with the passive view, and the CTS signal from the intersection of the active view with the probing beam.

receiver A and the fast system on receiver B during the CTS measurement in discharge 29600 as well as noise in the calibration data for each system. The uncertainty is seen to be dominated by noise in the spectra measured with the fast system on receiver B during the discharge. Noise in the calibration data for the fast system is significant only for frequencies near the stop band of the notch filters while noise in the filter bank measurements makes only a small contribution (due to the higher bit-resolution and lower overall signal level in the passive view). The lower panel of Fig. 11 shows the signal-tonoise ratio using the combined uncertainty for the spectrum in Fig. 9. For the frequency range corresponding to the bulk-ion region of CTS spectra the signal-to-noise ratio reaches values around 10-40, indicating that the bulk-ion region of the spectrum can in fact be resolved with this technique. However, fast-ion contributions to the CTS spectrum, which typically amount to a few $\mathrm{eV}$ at frequency shifts beyond $0.5 \mathrm{GHz}$, would be comparable to the noise level of the measurements with the fast receiver system. Fast-ion measurements with the fast receiver system would therefore require longer integration times or a significant noise level reduction.

\section{EXAMPLES OF MEASURED BULK ION CTS SPECTRA}

Discharge 29600 was designed for fast-ion studies, so the heating scheme was varied to include phases with ECRH heating alone as well as phases with NBI sources Q3 and Q8 individually and combined. Figure 12 shows individual examples of CTS spectra measured for different heating powers. As expected, the CTS spectra become broader at higher heating power and plasma temperature. Close inspection further shows a small positive shift in the spectra of about 15$20 \mathrm{MHz}$ indicating the presence of plasma rotation. As a
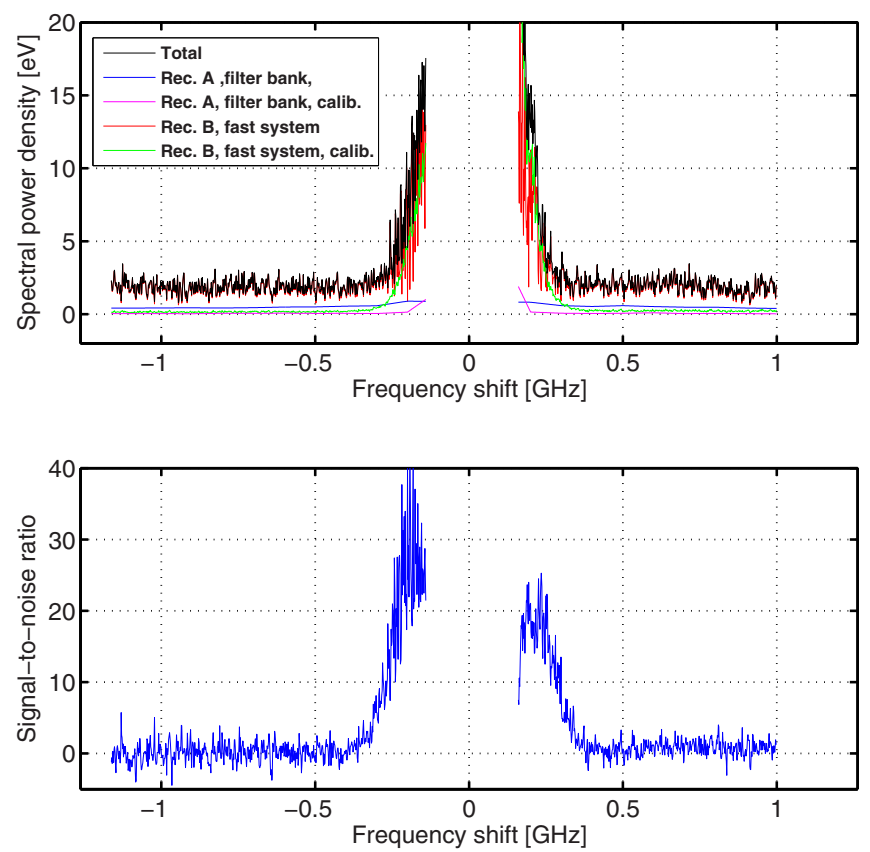

FIG. 11. Statistical uncertainty and signal-to-noise ratio for the spectrum shown in Fig. 9. The top panel shows the total uncertainty (black line) calculated from the standard deviation of fluctuations in the measurement and calibration data. This includes contributions from fluctuations in the spectra measured during the plasma discharge with the filter bank on receiver A (blue line) and in the calibration data for that system (magenta line) as well as fluctuations in the spectra measured during the plasma discharge with the fast system on receiver B (red line) and in the corresponding calibration data for that system (green line). The lower panel shows the combined signal-tonoise-ratio, which reaches values in the range 10-40 in the frequency range with significant bulk-ion related CTS signal.

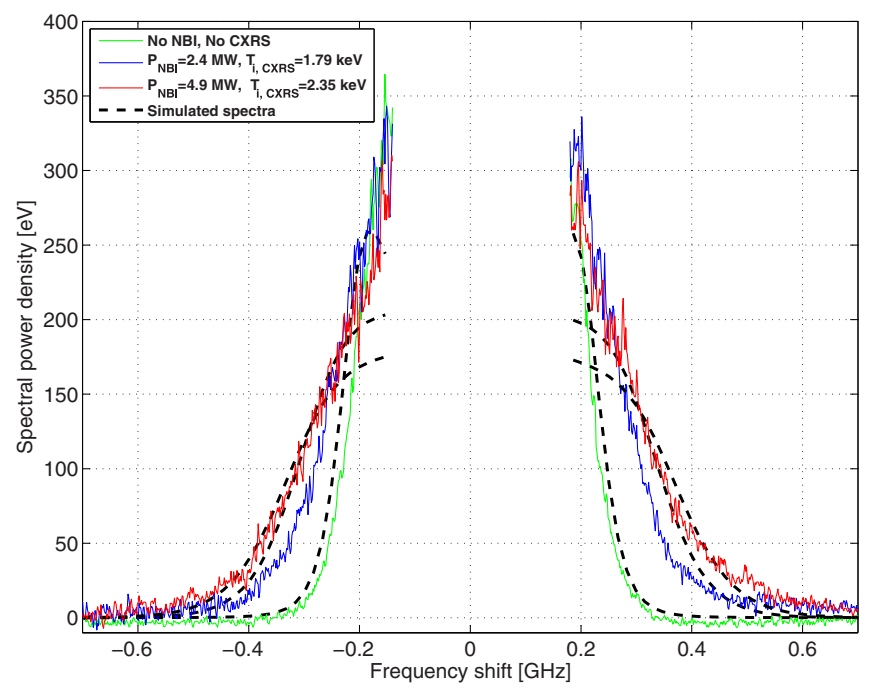

FIG. 12. Examples of spectra measured in AUG discharge 29600 with different NBI powers: at $t=2.38 \mathrm{~s}$ with no NBI (green), at $t=2.10 \mathrm{~s}$ with NBI source Q3 alone (blue), and at $t=3.43 \mathrm{~s}$ with Q3 and Q8 combined (red). The spectra were measured with the scattering geometry illustrated in Figs. 2 and 10 , and they represent data averaged over one gyrotron pulse each. Data from the frequency range affected by the notch filters are excluded. When available, the ion temperatures measured by CXRS are indicated in the legend. The response of the CTS spectra to the changing heating power and plasma temperature can be distinguished above the noise with the spectra clearly seen to become wider at higher heating power. A small positive frequency shift of $15-20 \mathrm{MHz}$ in the spectra also indicates the presence of plasma rotation during phases with NBI heating. The black dashed lines show simulated spectra as discussed in the text. 
consistency check, the dashed lines in Fig. 12 show spectra simulated with a theoretical model for CTS. ${ }^{1}$ Here, we use the ion temperatures and rotation velocities measured by charge exchange recombination spectrocopy (CXRS) as input when such measurements were available. For the spectrum with no NBI heating, when CXRS was unavailable, we use guessed values for the ion temperature and rotation velocity $\left(T_{i}\right.$ $=700 \mathrm{eV}, V_{i}=0$ ). The electron density and temperature were obtained from incoherent Thomson scattering, the magnetic field from equilibrium reconstruction, and the angles characterizing the scattering geometry from raytracing. Impurities affect the spectrum mainly at frequencies in or near the notch filter stop band, here roughly within $\pm 250 \mathrm{MHz}$. We have included helium, boron, carbon, and tungsten at concentration levels indicated by spectroscopic measurements (3\%, $0.3 \%, 0.3 \%$, and $0.1 \%$ of the electron density, respectively). The simulated and measured spectra are in reasonable agreement considering that the simulations are based on forward modelling alone with no fitting involved. Better agreement can be obtained by fitting the model to the data with some (or even all) of the model parameters considered free fitting parameters, and with prior knowledge about these parameters and their measurement uncertainties included through Bayesian methods. ${ }^{53}$ However, such an advanced treatment goes beyond the scope of the analysis we will present here.

In discharge 29600, the CTS scattering geometry was arranged with a projection angle, $\phi=\angle\left(\mathbf{k}^{\delta}, \mathbf{B}\right)=107^{\circ}$. Here, $\mathbf{k}^{\delta}=\mathbf{k}^{\mathrm{s}}-\mathbf{k}^{\mathrm{i}}$ is the difference between the scattered, $\mathbf{k}^{\mathrm{s}}$, and incident, $\mathbf{k}^{\mathrm{i}}$, radiation wave vectors. $\mathbf{k}^{\delta}$ also defines the wave vector of the plasma fluctuations resolved by the CTS measurement and thereby the direction of the 1D-projection of the ion velocity distribution which can be inferred from the measurements. While this is more relevant for fast-ion than bulk ion measurements, we note that in discharge 29600 the CTS measurements were thus more sensitive to velocity components perpendicular to the magnetic field than to parallel velocity components. ${ }^{2,3,39}$ Figure 13 shows an example of a spectrogram obtained in AUG discharge 30063 for a projection angle even closer to perpendicular, $\phi=92.4^{\circ}$. Here, the receiver beam was swept across the probing beam by changing the receiver mirror elevation angle in order to verify the position of the optimal beam overlap. The CTS signal intensity then gradually increases and decreases as the beam overlap first grows and then decays away. The optimal overlap position was found at $t=1.73 \mathrm{~s}$. From raytracing calculations, the measurement volume was then $13 \mathrm{~cm}$ across and centered on $(R, z)=(1.57 \mathrm{~m},-0.19 \mathrm{~m})$ corresponding to a normalized poloidal flux coordinate of 0.47 . We note that this is about $3 \mathrm{~cm}$, or $1.2^{\circ}$ in terms of the mirror elevation angle, away from the theoretically expected position of the maximum overlap as found by ray tracing. This difference is less than the size of the overlap itself. The ECRH mirror alignment is expected to be accurate within $0.1^{\circ}$ with the coupling of the receiver beam pattern to the ECRH waveguides contributing additional minor uncertainties to the beam alignment. Apart from such uncertainties, the difference therefore likely results mostly from uncertainties in the ray tracing calculation as this depends on measurements of the magnetic equilibrium and electron den-

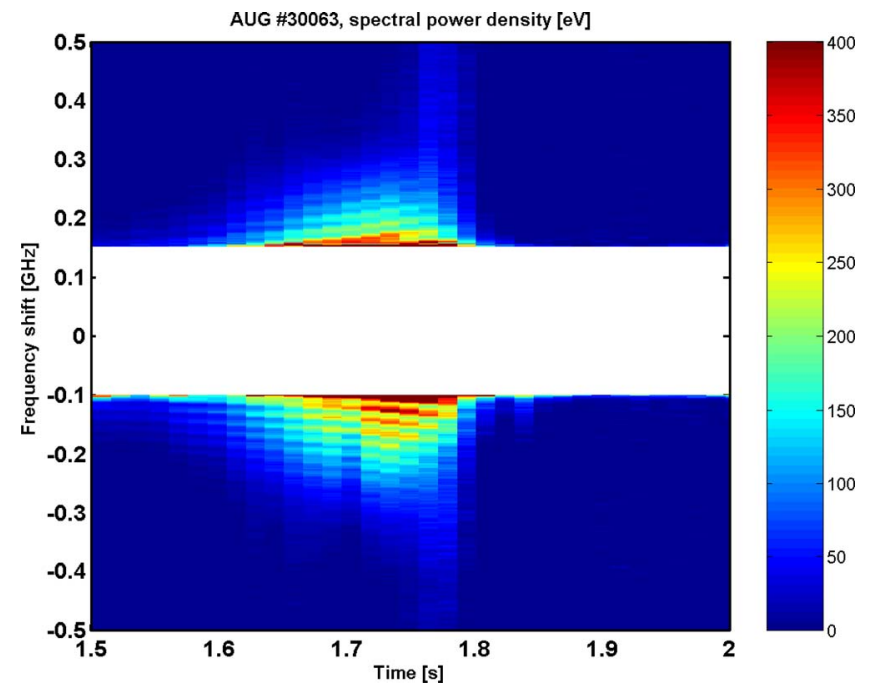

FIG. 13. A spectrogram of an overlap sweep conducted in AUG discharge 30063. The spectra are averaged over one gyrotron pulse giving a time resolution of $5 \mathrm{~ms}$. Data from the frequency range affected by the notch filters are excluded. The receiver beam is swept across the probing beam to identify the optimal overlap position where the signal is maximized. For the scattering geometries used here, with $\phi=92.4^{\circ}$ at the maximum overlap, the effects of the ion cyclotron motion and weakly damped ion Bernstein waves create peaks in the CTS spectra, which here appear as stripes in the spectrogram - see also Fig. 14 for individual spectra. The measurement location and the value of $\phi$ change slightly during the overlap sweep resulting in a small, gradual shift in the peak location during the sweep.

sity profile and is particularly sensitive to the edge density gradient, which was not measured in this discharge.

As also seen in Fig. 14, the bulk ion region of the CTS spectra now contains a sequence of peaks, which appear as stripes in the spectrogram. These so-called ion cyclotron structures, which appear for resolved wave vectors near perpendicular to the magnetic field, $\phi \simeq 90^{\circ}$, are signatures of the ion cyclotron motion and of weakly damped ion Bernstein waves exited by the ion motion. ${ }^{36}$ They appear at frequency intervals corresponding to the cyclotron frequencies of the main ion species present in the plasma-i.e., at intervals of about $20 \mathrm{MHz}$ for discharge 30063, which was deuterium fueled. The shape and relative size of peaks in the ion cyclotron structures are highly sensitive to the density ratios of the corresponding ion species. Measurements of ion cyclotron structures were demonstrated as a plasma composition and ion temperature diagnostic on TEXTOR ${ }^{30-33}$ and have been suggested as a possible fuel ion ratio diagnostic for ITER ${ }^{35,42}$ Resolving these features in the CTS spectra is therefore an important goal for the fast receiver system on AUG.

Figure 14 compares CTS spectra from discharges with different plasma compositions. The spectrum from discharge 30063 was measured in a deuterium fuelled plasma at the maximum overlap position during the overlap sweep. The peaks are separated by about $20 \mathrm{MHz}$ as expected for the deuterium ion cyclotron frequency at the location of the CTS measurement volume with a local magnetic field strength of 2.7 T. The spectrum from discharge 30122 was measured in a hydrogen fueled plasma using the scattering geometry established by the overlap sweep in discharge 30063 . The peaks now appear with a separation of roughly $40 \mathrm{MHz}$ as expected 


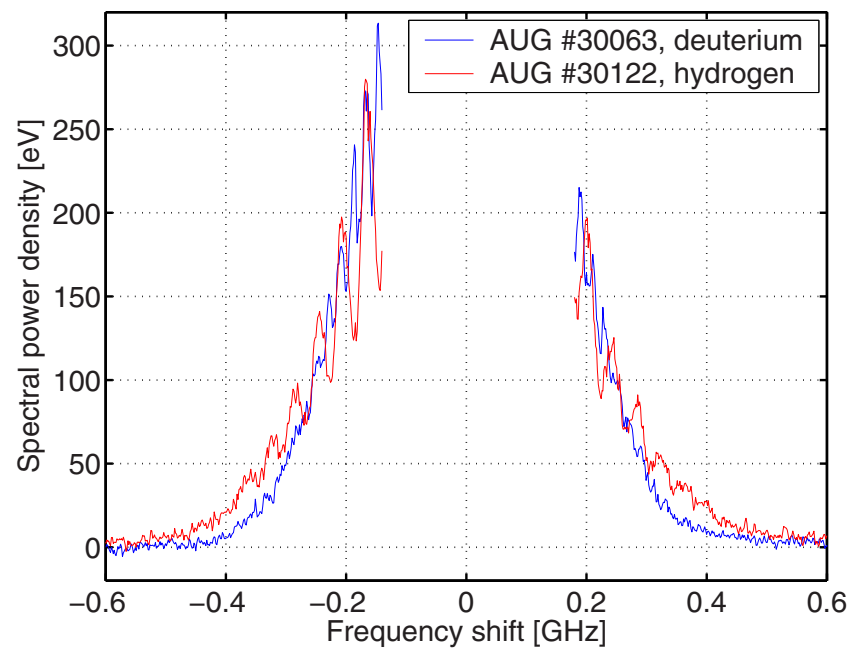

FIG. 14. Examples of CTS spectra with ion cyclotron structures measured in discharges dominated by deuterium (30063, at $t=1.73 \mathrm{~s})$ and hydrogen (30122, at $t=1.95 \mathrm{~s}$ ), respectively. The spectra represent data averaged over one gyrotron pulse each. Data from the frequency range affected by the notch filters are excluded. In response to the different fueling schemes, the peaks in the ion cyclotron structures change shape and frequency interval. In discharge 30063 , they appear at intervals of about $20 \mathrm{MHz}$ corresponding to the deuterium ion cyclotron frequency in the CTS measurement volume. In discharge 30122, they appear at intervals of $40 \mathrm{MHz}$ corresponding to the hydrogen ion cyclotron frequency.

for the hydrogen ion cyclotron frequency. The fast acquisition system can thus resolve the ion cyclotron structures and distinguish the effects of different fueling schemes on the plasma composition. Detailed interpretation of the measured spectra to infer the actual plasma temperature and composition requires fitting with a theoretical model for the CTS spectrum, ${ }^{30,32,33}$ which is beyond the scope of the work presented here.

\section{CONCLUSION AND OUTLOOK}

The bulk ion region of CTS spectra is of interest for its diagnostic potential regarding measurements of the composition, ion temperature, and rotation velocity of fusion plasmas. To access this information, we have equipped the CTS system on AUG with a new fast acquisition system enabling measurements with a frequency resolution in the range of $1 \mathrm{MHz}$ as required to properly resolve the bulk ion region of CTS spectra. Here, we have discussed the design, calibration, and operation of the fast acquisition system, and we have shown examples of the first bulk ion measurements demonstrating the ability of the system to resolve the required features in the CTS spectrum as well as their sensitivity to changes in plasma heating and fueling.

Presently, the total measurement time of the fast acquisition system is limited to $160 \mathrm{~ms}$ per discharge by the amount of memory available on the digitizer. To relax this constraint, the system is being upgraded to enable data to be streamed directly to a larger memory bank using a first-in-first-out (FIFO) protocol. The upgraded system achieves transfer rates up to $800 \mathrm{MB} / \mathrm{s}$ when not acquiring data and about $550 \mathrm{MB} / \mathrm{s}$ during data acquisition. In the future, this will allow the acquisition of data sets up to $4.8 \mathrm{~GB}$ over the course of a $7 \mathrm{~s}$ long
AUG discharge. Assuming a sampling rate of $6.25 \mathrm{GS} / \mathrm{s}$ this would correspond to a total measurement time of $0.78 \mathrm{~s}$ or a maximum duty cycle of $11 \%$, up from previously $2 \%$. This increase in acquisition time can be used to improve the signalto-noise ratio for individual measurements by lengthening the acquisition time per gyrotron pulse, or it can be used to cover a larger part of each plasma discharge. In the long term, realtime FFT processing and averaging of the data using fieldprogrammable gate arrays ${ }^{54}$ will enable near-continuous coverage and may allow real-time analysis of the spectra to infer plasma parameters such as the ion temperature and rotation velocity. Work is now underway to implement such a system in one of the CTS receivers on AUG.

\section{ACKNOWLEDGMENTS}

This work has been supported by the European Communities under the contract of Association between EURATOM and DTU. It was carried out within the framework of the European Fusion Development Agreement under EFDA Contract No. WP13-IPH-A10-P1-01. The views and opinions expressed herein do not necessarily reflect those of the European Commission.

${ }^{1}$ H. Bindslev, J. Atmos. Terr. Phys. 58, 983 (1996).

${ }^{2}$ M. Salewski, B. Geiger, S. Nielsen, H. Bindslev, M. García-Muñoz, W. Heidbrink, S. Korsholm, F. Leipold, F. Meo, P. Michelsen, D. Moseev, M. Stejner, and G. Tardini, Nucl. Fusion 52, 103008 (2012).

${ }^{3}$ M. Salewski, S. Nielsen, H. Bindslev, V. Furtula, N. N. Gorelenkov, S. B. Korsholm, F. Leipold, F. Meo, P. K. Michelsen, D. Moseev, and M. Stejner, Nucl. Fusion 51, 083014 (2011).

${ }^{4}$ H. Bindslev, J. Hoekzema, J. Egedal, J. Fessey, T. Hughes, and J. Machuzak, Phys. Rev. Lett. 83, 3206 (1999).

${ }^{5}$ H. Bindslev, S. Nielsen, L. Porte, J. Hoekzema, S. B. Korsholm, F. Meo, P. K. Michelsen, S. Michelsen, J. Oosterbeek, E. Tsakadze, E. Westerhof, and P. Woskov, Phys. Rev. Lett. 97, 205005 (2006).

${ }^{6}$ H. Bindslev, S. K. Nielsen, L. Porte, J. A. Hoekzema, S. B. Korsholm, F. Meo, P. K. Michelsen, S. Michelsen, J. W. Oosterbeek, E. L. Tsakadze, E. Westerhof, and P. Woskov, Plasma Phys. Controlled Fusion 49, B551 (2007).

${ }^{7}$ S. K. Nielsen, H. Bindslev, L. Porte, J. A. Hoekzema, S. B. Korsholm, F. Leipold, F. Meo, P. K. Michelsen, S. Michelsen, J. W. Oosterbeek, E. L. Tsakadze, G. Van Wassenhove, E. Westerhof, and P. Woskov, Phys. Rev. E 77, 016407 (2008)

${ }^{8}$ S. K. Nielsen, H. Bindslev, M. Salewski, A. Bürger, E. Delabie, V. Furtula, M. Kantor, S. B. Korsholm, F. Leipold, F. Meo, P. K. Michelsen, D. Moseev, J. W. Oosterbeek, M. Stejner, E. Westerhof, and P. Woskov, Plasma Phys. Controlled Fusion 52, 092001 (2010).

${ }^{9}$ M. Salewski, F. Meo, M. Stejner, O. Asunta, H. Bindslev, V. Furtula, S. B. Korsholm, T. Kurki-Suonio, F. Leipold, F. Leuterer, P. K. Michelsen, D. Moseev, S. K. Nielsen, J. Stober, G. Tardini, D. Wagner, and P. Woskov, Nucl. Fusion 50, 035012 (2010).

${ }^{10}$ F. Meo, M. Stejner, M. Salewski, H. Bindslev, T. Eich, V. Furtula, S. B. Korsholm, F. Leuterer, F. Leipold, P. K. Michelsen, D. Moseev, S. K. Nielsen, B. Reiter, J. Stober, D. Wagner, P. Woskov, and T. A. U. Team, J. Phys.: Conf. Ser. 227, 012010 (2010).

${ }^{11}$ S. K. Nielsen, M. Salewski, H. Bindslev, A. Bürger, V. Furtula, M. Kantor, S. B. Korsholm, H. R. Koslowski, A. Krämer-Flecken, F. Leipold, F. Meo, P. K. Michelsen, D. Moseev, J. W. Oosterbeek, M. Stejner, and E. Westerhof, Nucl. Fusion 51, 063014 (2011).

${ }^{12}$ D. Moseev, F. Meo, S. B. Korsholm, T. Koskela, M. Albergante, O. Asunta, H. Bindslev, A. Bürger, V. Furtula, M. Y. Kantor, F. Leipold, P. K. Michelsen, S. K. Nielsen, M. Salewski, O. Schmitz, M. Stejner, and E. Westerhof, Plasma Phys. Controlled Fusion 53, 105004 (2011).

${ }^{13}$ M. Nishiura, S. Kubo, K. Tanaka, N. Tamura, T. Shimozuma, T. Mutoh, K. Kawahata, T. Watari, T. Saito, Y. Tatematsu, T. Notake, and L. Experiment Group, J. Phys.: Conf. Ser. 227, 012014 (2010). 
${ }^{14}$ M. Nishiura, S. Kubo, K. Tanaka, R. Seki, S. Ogasawara, T. Shimozuma, K. Okada, S. Kobayashi, T. Mutoh, K. Kawahata, T. Watari, T. Saito, Y. Tatematsu, S. Korsholm, and M. Salewski, Nucl. Fusion 54, 023006 (2014).

${ }^{15} \mathrm{H}$. Bindslev, F. Meo, and S. B. Korsholm, "ITER fast ion collective Thomson scattering - Feasibility study," Technical Report, 2003, see http:// orbit.dtu.dk/en/publications/iter-fast-ion-collective-thomson-scattering (401e562c-65a3-4345-9032-da5cc8b9013b).html.

${ }^{16}$ F. Meo, H. Bindslev, S. B. Korsholm, E. L. Tsakadze, C. I. Walker, P. Woskov, and G. Vayakis, Rev. Sci. Instrum. 75, 3585 (2004).

${ }^{17}$ M. Salewski, L.-G. Eriksson, H. Bindslev, S. Korsholm, F. Leipold, F. Meo, P. Michelsen, and S. Nielsen, Nucl. Fusion 49, 025006 (2009).

${ }^{18}$ M. Salewski, O. Asunta, L.-G. Eriksson, H. Bindslev, V. Hynönen, S. B. Korsholm, T. Kurki-Suonio, F. Leipold, F. Meo, P. K. Michelsen, S. K. Nielsen, and J. Roenby, Plasma Phys. Controlled Fusion 51, 035006 (2009).

${ }^{19}$ S. Korsholm, H. Bindslev, V. Furtula, F. Leipold, F. Meo, P. Michelsen, D. Moseev, S. Nielsen, M. Salewski, and M. Stejner, Nucl. Instrum. Methods Phys. Res. A 623, 677 (2010).

${ }^{20}$ P. John, Phys. Rev. A 6, 756 (1972).

${ }^{21}$ G. Wurden, M. Ono, and K. Wong, Phys. Rev. A 26, 2297 (1982).

${ }^{22}$ H. Park, P. S. Lee, W. A. Peebles, and N. C. Luhmann, Rev. Sci. Instrum. 56, 922 (1985).

${ }^{23}$ R. Behn, D. Dicken, J. Hackmann, S. Salito, M. Siegrist, P. Krug, I. Kjelberg, B. Duval, B. Joye, and A. Pochelon, Phys. Rev. Lett. 62, 2833 (1989).

${ }^{24}$ F. Orsitto, Rev. Sci. Instrum. 63, 4651 (1992).

${ }^{25}$ F. Orsitto, Rev. Sci. Instrum. 61, 3093 (1990).

${ }^{26}$ M. R. Siegrist, R. Behn, D. Dicken, B. P. Duval, J. Hackmann, B. Joye, J. Lister, C. Nieswand, A. Pochelon, G. Soumagne, and D. C. Yuan, J. Appl. Phys. 69, 1993 (1991).

${ }^{27}$ M. Born, H. D. Dicken, J. Hackmann, and J. Uhlenbusch, Plasma Phys. Controlled Fusion 35, 391 (1993).

${ }^{28}$ E. V. Suvorov, V. Erckmann, E. Holzhauer, W. Kasparek, Y. A. Dryagin, S. E. Fil'chenkov, A. A. Fraiman, T. Geist, M. Kick, L. M. Kukin, A. V. Kostrov, L. V. Lubyako, A. M. Shtanyuk, N. K. Skalyga, and O. B. Smolyakova, Plasma Phys. Controlled Fusion 37, 1207 (1995).

${ }^{29}$ E. V. Suvorov, E. Holzhauer, W. Kasparek, L. V. Lubyako, A. B. Burov, Y. A. Dryagin, S. E. Fil'chenkov, A. A. Fraiman, L. M. Kukin, A. V. Kostrov, D. A. Ryndyk, A. M. Shtanyuk, N. K. Skalyga, O. B. Smolyakova, V. Erckmann, T. Geist, M. Kick, H. Laqua, and M. Rust, Plasma Phys. Controlled Fusion 39, B337 (1997).

${ }^{30}$ M. Stejner, M. Salewski, S. B. Korsholm, H. Bindslev, E. Delabie, F. Leipold, F. Meo, P. K. Michelsen, D. Moseev, S. K. Nielsen, A. Bürger, and M. de Baar, Plasma Phys. Controlled Fusion 55, 085002 (2013).

${ }^{31}$ S. B. Korsholm, M. Stejner, H. Bindslev, V. Furtula, F. Leipold, F. Meo, P. Michelsen, D. Moseev, S. Nielsen, M. Salewski, M. de Baar, E. Delabie, M. Kantor, and A. Bürger, Phys. Rev. Lett. 106, 165004 (2011).

${ }^{32}$ M. Stejner, S. B. Korsholm, S. K. Nielsen, M. Salewski, H. Bindslev, S. Brezinsek, V. Furtula, F. Leipold, P. K. Michelsen, F. Meo, D. Moseev, A. Bürger, M. Kantor, and M. de Baar, Plasma Phys. Controlled Fusion 54, 015008 (2012).

${ }^{33}$ M. Stejner, S. B. Korsholm, S. K. Nielsen, M. Salewski, H. Bindslev, F. Leipold, P. K. Michelsen, F. Meo, D. Moseev, A. Büger, M. Kantor, and M. de Baar, Rev. Sci. Instrum. 83, 10 E307 (2012).

${ }^{34}$ J. S. Ross, H.-S. Park, P. Amendt, L. Divol, N. L. Kugland, W. Rozmus, and S. H. Glenzer, Rev. Sci. Instrum. 83, 10E323 (2012).

${ }^{35}$ M. Stejner, S. B. Korsholm, S. K. Nielsen, M. Salewski, H. Bindslev, V. Furtula, F. Leipold, P. K. Michelsen, F. Meo, and D. Moseev, Nucl. Fusion 52, 023011 (2012).

${ }^{36}$ M. Stejner, S. K. Nielsen, H. Bindslev, S. B. Korsholm, and M. Salewski, Plasma Phys. Controlled Fusion 53, 065020 (2011).

${ }^{37}$ A. Herrmann, and O. Gruber, Fusion Sci. Technol. 44, 569 (2003).

${ }^{38}$ B. Streibl, P. T. Lang, F. Leuterer, J.-M. Noterdaeme, and A. Stäbler, Fusion Sci. Technol. 44, 578 (2003).
${ }^{39}$ S. K. Nielsen, M. Stejner, J. Rasmussen, A. S. Jacobsen, S. B. Korsholm, F. Leipold, M. Maraschek, F. Meo, P. K. Michelsen, D. Moseev, M. Salewski, M. Schubert, J. Stober, W. Suttrop, G. Tardini, and D. Wagner, "Measurements of the fast-ion distribution function at ASDEX Upgrade by collective Thomson scattering (CTS) using active and passive views," Plasma Phys. Controlled Fusion (to be published).

${ }^{40}$ J. Rasmussen, S. K. Nielsen, M. Stejner, M. Salewski, A. S. Jacobsen, S. B. Korsholm, F. Leipold, F. Meo, P. K. Michelsen, D. Moseev, M. Schubert, J. Stober, G. Tardini, D. Wagner, and ASDEX Upgrade, AIP Conf. Proc. 1612, 117 (2014).

${ }^{41}$ H. Bindslev, F. Meo, E. L. Tsakadze, S. B. Korsholm, and P. Woskov, Rev. Sci. Instrum. 75, 3598 (2004).

${ }^{42}$ H. Bindslev, A. W. Larsen, F. Meo, P. Michelsen, S. Michelsen, A. H. Nielsen, S. Nimb, and E. Tsakadze, "ITER fast ion collective Thomson scattering: Detailed integrated design of the collective Thomson scattering (CTS) system for ITER," Technical Report, 2005, see http://orbit.dtu.dk/en/ publications/iter-fast-ion-collective-thomson-scattering(220dc911-ddcb43a5-9f96-c7a2a0153f86).html.

${ }^{43}$ F. Meo, H. Bindslev, and S. B. Korsholm, "ITER fast ion collective Thomson scattering: Conceptual design of $60 \mathrm{GHz}$ system, Ris $\varnothing-\mathrm{R}-1600(\mathrm{EN}), "$ Technical Report, 2007, see http://www.orbit.dtu.dk/en/publications/ iter-fast-ion-collective-thomson-scattering-conceptual-design-of-60-ghzsystem(30e4aab5-22c1-43e0-91a1-39f12584b782).html.

${ }^{44}$ D. Wagner, G. Grünwald, F. Leuterer, A. Manini, F. Monaco, M. Münich, H. Schütz, J. Stober, H. Zohm, T. Franke, M. Thumm, G. Gantenbein, R. Heidinger, A. Meier, W. Kasparek, C. Lechte, A. Litvak, G. Denisov, A. Chirkov, E. Tai, L. Popov, V. Nichiporenko, V. Myasnikov, E. Solyanova, S. Malygin, F. Meo, and P. Woskov, Nucl. Fusion 48, 054006 (2008).

${ }^{45}$ D. Wagner, J. Stober, F. Leuterer, G. Sips, G. Grunwald, F. Monaco, M. Munich, E. Poli, H. Schutz, F. Volpe, W. Treutterer, H. Zohm, T. Franke, M. Thumm, R. Heidinger, G. Gantenbein, A. Meier, W. Kasparek, C. Lechte, A. Litvak, G. Denisov, A. Chirkov, E. Tai, L. Popov, V. Nichiporenko, V. Myasnikov, E. Solyanova, and S. Malygin, IEEE Trans. Plasma Sci. 37, 395 (2009).

${ }^{46}$ J. Stober, A. Bock, H. Höhnle, M. Reich, F. Sommer, W. Treutterer, D. Wagner, L. Gianone, A. Herrmann, F. Leuterer, F. Monaco, M. Marascheck, A. Mlynek, S. Müller, M. Münich, E. Poli, M. Schubert, H. Schütz, H. Zohm, W. Kasparek, U. Stroth, A. Meier, T. Scherer, D. Strau $\beta$, A. Vaccaro, J. Flamm, M. Thumm, A. Litvak, G. Denisov, A. Chirkov, E. Tai, L. Popov, V. Nichiporenko, V. Myasnikov, E. Soluyanova, and S. Malygin, EPJ Web Conf. 32, 02011 (2012).

${ }^{47}$ F. Meo, H. Bindslev, S. B. Korsholm, V. Furtula, F. Leuterer, F. Leipold, P. K. Michelsen, S. K. Nielsen, M. Salewski, J. Stober, D. Wagner, and P. Woskov, Rev. Sci. Instrum. 79, 10E501 (2008).

${ }^{48}$ V. Furtula, M. Salewski, F. Leipold, P. K. Michelsen, S. B. Korsholm, F. Meo, D. Moseev, S. K. Nielsen, M. Stejner, and T. Johansen, Rev. Sci. Instrum. 83, 013507 (2012).

${ }^{49}$ L. Porte, H. Bindslev, F. Hoekzema, J. Machuzak, P. Woskov, and D. Van Eester, Rev. Sci. Instrum. 72, 1148 (2001).

${ }^{50}$ S. Michelsen, S. B. Korsholm, H. Bindslev, F. Meo, P. K. Michelsen, E. L. Tsakadze, J. Egedal, P. Woskov, J. A. Hoekzema, F. Leuterer, and E. Westerhof, Rev. Sci. Instrum. 75, 3634 (2004).

${ }^{51}$ M. Stejner, S. K. Nielsen, S. B. Korsholm, M. Salewski, H. Bindslev, V. Furtula, F. Leipold, F. Meo, P. K. Michelsen, D. Moseev, A. Bürger, M. Kantor, and M. de Baar, Rev. Sci. Instrum. 81, 10D515 (2010).

${ }^{52}$ P. Woskov, H. Bindslev, F. Leipold, F. Meo, S. K. Nielsen, E. L. Tsakadze, S. B. Korsholm, J. Scholten, C. Tito, E. Westerhof, J. W. Oosterbeek, F. Leuterer, F. Monaco, M. Muenich, and D. Wagner, Rev. Sci. Instrum. 77, 10E524 (2006).

${ }^{53}$ H. Bindslev, Rev. Sci. Instrum. 70, 1093 (1999).

${ }^{54}$ W. A. Bongers, V. van Beveren, D. J. Thoen, P. J. W. M. Nuij, M. R. de Baar, A. J. H. Donné, E. Westerhof, A. P. H. Goede, B. Krijger, M. A. van den Berg, M. Kantor, F. Graswinckel, M. B. A. Hennen, and F. C. Schüller, Rev. Sci. Instrum. 82, 063508 (2011). 